\title{
EDITORIAL
}

\section{Gene Editing: Practical Application in the Lab}

\author{
Aron M. Geurts ${ }^{1}$ and Stephen C. Ekker ${ }^{2}$ \\ ${ }^{1}$ Medical College of Wisconsin, Milwaukee, Wisconsin; ${ }^{2}$ Mayo Clinic, Rochester, Minnesota.
}

The PRACTICAL IMPLEMENTATION of emergent technology in research laboratories both large and small is a constant challenge as investigators at all stages seek to remain competitive in the current researchfunding environment. Arguably, genome editingusing CRISPR/Cas9, TALEN, and ZFNs-and its many variant themes represents a proliferative, impactful, and widely useful technological advance spanning the spectrum of genetic research and phylogeny of model systems unwitnessed since the description of the polymerase chain reaction. The pervasive spread of these technologies penetrates even our pocketbooks as peer reviewers demand to see these innovative approaches in competitive grant applications and renewals, with preliminary data to boot, further encouraging the rapid uptake of these tools and protocols. Investigators are quickly met with a pressing need to find an experienced collaborator or attempt to implement tools and protocols on their own that are intricate and often unfamiliar. Luckily, there has been no shortage of access to the basic reagents so generously provided by the innovators of these technologies through repositories at negligible cost and restriction to academic researchers, and the explosion of commercial products. Yet, despite this accessibility, some important practical thinking and specific skillsets are necessary to craft the appropriate approach and tools for the job at hand. This special issue presents five articles describing practical improvements and guidance in implementing genome editing protocols in your laboratory, from improved reagents and more reproducible protocols for construction of customs reagents, either one at a time or in massive parallel, to simple and scalable protocols for detection of specific genome editing events, and finally, deployment of these tools in cell and animal model systems.
Since the initial description of the assembly of the modular transcriptional activator-like (TAL) domain repeats for TALEN construction via the Golden Gate cloning method, incremental improvements have been described to shorten the required number of steps and reduce the number of plasmids necessary to propagate the TAL libraries. Here, Welker et al. ${ }^{1}$ identify a design flaw in one of the original TALEN Golden Gate vectors, especially popular among researchers for rapid construction of reagents for zebrafish mutagenesis, which can lead to recovery of undesirable recombinants during the final cloning reaction. A quick fix to the vector is described, resulting in highly efficient and reproducible recovery of desired clones. The Ekker lab takes scalable assembly of TALENs a step further, reducing the hands-on time for construction of new reagents to a single-day, single-tube reaction via the FusX system. $^{2}$ Furthermore, the FusX method of TALEN construction can be automated using liquid handling robots and simple inventory management programming that won't break the laboratory budget.

In the CRIPSR/Cas9 world, custom reagent construction is not a limiting factor, but effective delivery of the appropriate dosage of the Cas9 enzyme and short guide RNAs (sgRNAs) can be challenging in some systems. Single- and dualexpression systems are widely popular to deliver these components and come in many flavors, with various tags and selectable markers. However, plasmid delivery is not feasible or desirable in some approaches, and so vectors and protocols for in vitro transcription (IVT) of both Cas9 mRNA and sgRNAs are widely used. More recently, purified recombinant Cas9 is gaining popularity and becoming widely commercially available with certified activity. When combined with IVT-generated sgRNAs, the ribonuclear protein (RNP) complex is poised for 
rapid and transient mutagenesis upon delivery to the cell nucleus and finding its target gene. Here, Kouranova et al. $^{3}$ compare these various delivery methods in rodent cells and embryos for production of mutant animals and find that RNP is a stable, reliable, and controllable method in these systems. They further provide important advice for accurate quantitation of sgRNAs produced via the IVT reaction.

One of the initially surprising challenges for implementing genome editing tools in various applications can be the difficulty in detecting the desired sequence event, which can be a rare occurrence among many unmodified or undesirable DNA-repair modified cells or individuals in a population. Many gene edit designs will seek to insert or destroy an endonuclease recognition sequence, allowing for genotyping of individuals via restriction fragment length polymorphism (RFLP) analysis. While often not the primary edit of significance in the experiment, these types of modifications can sometimes be incorporated as silent mutations as not to further perturb gene function. Commercially designed sequence-specific fluorescent probes are well vetted for efficient and reproducible detection of even single variants but can be quite expensive for the small laboratory needing to rapidly screen large numbers of individuals for the mutant of interest. Lee et al. ${ }^{4}$ describe an exciting and simple open-source allele-specific quantitative (ASQ) PCR method of genotyping using universal fluorescent probes. The ASQ-PCR method demonstrates reliable performance against peer methods and will likely be adopted by many and further refined as a scalable and cost-effective approach to detect individuals harboring specific engineered events.

Finally, in an elegant example of cellular genome editing, Takata et al. describe herein a simple design method for inserting reporter genes into mouse stem cells and 3D tissue organoids, which they call GET (genome editing with targeting vector), further expanding the examples and utility of genome editing in mouse stem cell systems. Their approach was complemented by transposon-based RNA interference (RNAi) to fine-tune inducible knockdown of a target gene, Fgf5, in a titratable and time-dependent fashion. The authors smartly propose such 3D culture systems as a potential for future studies of "developmental biology in a dish," where genes can be easily and quickly manipulated and signaling pathways analyzed. From RNAi to CRISPRs to TALENs, the articles in this special issue showcase the wonderful diversity of genome engineering approaches available to scientists today and awaken our imagination to what is still to come. We anticipate many more exciting systems and new creative approaches as this field of cellular programming embraces and continues to define some key signature science for the 21 st century.

\section{REFERENCES}

1. Welker JM, Wierson WA, Wang Y, et al. GoldyTALEN vectors with improved efficiency for golden gate TALEN assembly. Hum Gene Ther 2016;6:423-424.

2. Ma AC, McNulty MS, Poshusta TL, C, et al. FusX: A rapid one-step transcription activator-like effector assembly system for genome science. Hum Gene Ther 2016;6:451-463
3. Kouranova E, Forbes K, Zhao G, et al. CRISPRs for optimal targeting: Delivery of CRISPR components as DNA, RNA, and protein into cultured cells and single-cell embryos. Hum Gene Ther 2016:6:464-475.

4. Lee HB, Schwab TL, Koleilat A, et al. Allelespecific quantitative PCR for accurate, rapid, and cost-effective genotyping. Hum Gene Ther 2016; 6:425-435.

5. Takata N, Sakakura E, Kasukawa T, et al. Establishment of functional genomics pipeline in epiblast-like tissue by combining transcriptomic analysis and gene knockdown/knockin/knockout, using RNA interference and CRISPR/Cas9. Hum Gene Ther 2016;6:436-450. 